\title{
Criterios de usabilidad como facilitador en el diseño de experiencias turísticas
}

\section{Usability criteria as facilitator in the design of tourist experiences}

\author{
Claudia E. Llontop Diez* \\ Escuela Profesional de Turismo y Hotelería, \\ Universidad de San Martin de Porres, Perú
}

\section{Resumen}

Se integra el concepto de usabilidad al proceso del diseño de experiencias turísticas a partir de las características del servicio y los actores que intervienen en su conjunto, en un contexto donde la tecnología y la conectividad han cambiado la forma y los procesos de atención y generación del servicio turístico, integrando nuevas formas de interacción con dispositivos y formas combinadas en operaciones cotidianas del servicio turístico. La forma como interviene la tecnología nos hace reflexionar sobre cómo debemos abordar el diseño de nuevos productos y servicios, y cómo hacer que continúen vigentes en el mercado. En este trabajo se abordan algunas pautas de la usabilidad integrándolas a un nuevo concepto de diseño que lleva como engranaje la mejora continua y adaptaciones del concepto «usabilidad».

Palabras clave: usabilidad; facilitador; tecnología y conectividad; mejora continua; experiencia turística

\begin{abstract}
This article aims to incorporate usability into the process of designing tourist experiences, based on the characteristics of tourist service and intervening actors as a whole. This happens in a context where technology
\end{abstract}

Este es un artículo Open Access bajo la licencia Creative Commons Atribución-NoComercial-Compartirlgual 4.0

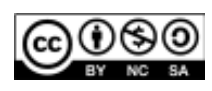


and connectivity have changed the modalities and processes of tourist service assistance and generation, by integrating new forms of interaction with devices and combined forms in tourist service daily operations. The way technology intervenes in this matter makes us reflect on how we should approach the design of new products and services, and how to keep them in force in the market. This paper addresses some usability guidelines by incorporating them into a new design that links together continuous improvement and adaptations of "usability".

Keywords: usability; facilitator; technology and connectivity; continuous improvement; tourist experience

\section{Introducción}

El turismo involucra una serie de actores que hacen posible la entrega del servicio al cliente. Los servicios y la atención que recibe un turista o visitante implica la operación de diferentes personas en las diversas fases del viaje o consumo de servicios turísticos.

En la actualidad es más fácil reservar un hotel, verificar su reputación y realizar diversas gestiones para ajustar la compra de un servicio a nuestros requerimientos mediante teléfonos móviles; las reservas pueden realizarse de manera rápida online así como su cancelación y forma de pago; podemos solicitar un taxi, establecer una ruta e indicarle volumen de equipaje, cantidad de personas que irán, si se lleva una silla de ruedas, entre otros. Los servicios de comida y delivery también se adaptan a la medida del cliente y nos proponen la facilidad de programar pedidos, todo gracias a la conectividad y el desarrollo de tecnologías que hacen posible esta realidad. No obstante, en la industria del turismo, un propósito importante, es la experiencia del cliente, que a buena cuenta, es quien se convierte en el «verdugo» frente a «malas propuestas» o servicios carentes de originalidad y uso.

Entender la forma cómo el cliente consume o hace uso de los servicios es fundamental. González-Sánchez, Montero-Simarro y Gutiérrez-Vela (2012), afirman al respecto: 
La calidad de la interacción influye de manera decisiva en que los usuarios se decanten por un sistema u otro. Los principales desafíos que presenta la definición de la calidad de la interacción en estos momentos están en la necesidad de considerar las propiedades hedónicas del proceso interactivo. (pp. 534-535)

En este sentido, caer en el desuso o ser obsoletos es uno de los factores que preocupan a los empresarios competitivos, así como, mantenerse en el mercado turístico. Las rutas tradicionales y los destinos caen en procesos de envejecimiento y declive por no entender al cliente o por desconocer la forma de usar sus productos. Por ello, la usabilidad extendida en el uso tecnológico de softwares nos abre las posibilidades de entender su integración al turismo y extender no solo la práctica a los sistemas que se emplean, sino también a procesos más complejos en un ámbito donde la conectividad y los servicios están íntimamente ligados y su empleo se hace más intensivo.

Estamos ante un cambio 'generacional' donde el objetivo no sólo está en que nuestros productos sean rápidos, eficaces y eficientes o la información esté bien diseñada y sea de fácil acceso. Se tiende a buscar ese valor añadido que es, que el usuario se sienta satisfecho (placer, felicidad, seguridad, fidelidad, etc.), provocando que la calidad del proceso de uso sea la mejor posible. (González-Sánchez et al., 2012, p. $535)$.

De este modo se hace más evidente la importancia del criterio de usabilidad en el diseño de servicios turísticos.

\section{Usabilidad y turismo}

La usabilidad es un concepto más completo del que se cree, comúnmente se entiende como «facilidad de uso», como resultado de interactuar con un sistema, producto o servicio. En un sentido más amplio el objetivo de diseñar y evaluar sistemas, productos y servicios enfocados en la usabilidad busca que los usuarios desarrollen un uso efectivo y eficiente, que se traduzca en satisfacción, teniendo en cuenta las circunstancias específicas en las que se utiliza un sistema, producto o servicio, así como el contexto de uso (NSAI Standard, 2019, p. 14). 
La usabilidad en el ámbito profesional destaca en el desarrollo de la tecnología, especialmente de softwares, pero en su sentido más amplio la usabilidad está considerada como «la medida en que un sistema, producto o servicio puede ser utilizado por usuarios específicos para lograr objetivos específicos con efectividad, eficiencia y satisfacción en un contexto específico de uso» (NSAI Standard, 2019, p. 14).

En un escenario, donde la tecnología forma parte de la vida y la actividad humana, es imposible imaginar la actividad turística sin el empleo de sistemas, dispositivos y conectividad. En las fases del ciclo de los viajes podemos observar como la tecnología y la conectividad interactúan con el visitante, ya sea para enterarse, informarse, ubicar, consultar, expresarse, elegir, tomar decisiones, disfrutar y compartir experiencias empleando diversos dispositivos, entre ellos, el Smartphone o teléfono inteligente.

En una nota de prensa la OMT Press Release (2019), anuncia que Google se asocia con la Organizacion Mundial del Turismo (OMT) para avanzar en el desarrollo de competencias digitales. Se afirma que las competencias digitales son de vital importancia para la competitividad de un sector como el nuestro, que se caracteriza por el cambio tecnológico y la creación constante de nuevos modelos de negocio. En este contexto, donde la tecnología y la conectividad intervienen en la industria de los viajes y los hábitos de los consumidores, Sakulsureeyadej (2011) refiere lo siguiente:

Dado que la tecnología está evolucionando más rápido que nunca, la mayoría de los viajeros de todo el mundo recurren a ella con mucha más frecuencia que en el pasado. Internet ha revolucionado la industria turística mucho más que cualquier otro factor en los últimos decenios. Asimismo, en vista de que cada vez más personas están conectadas entre sí, y tienen acceso a la abundante información que existe en línea, un número creciente de viajeros busca información por Internet antes de tomar cualquier decisión con respecto a sus viajes. (p. 9)

El contacto con redes sociales y la búsqueda de destinos se realiza hoy por smartphones, en el boletín $\mathrm{N}^{\circ} 54$ de Turismo In, publicado por PROMPERU se señala: 
Es difícil imaginar que hace apenas una década se viajaba sin las facilidades que proporcionan las nuevas tecnologías de telefonía móvil. Hoy, el 98\% de los viajeros llevan consigo sus smartphones durante sus experiencias turísticas. Desde el punto de vista de la oferta, es innegable que muchos procesos como la atención, la adquisición, la fidelización o la satisfacción del cliente se han trasladado a las pantallas de los dispositivos tecnológicos. Para el futuro de corto y mediano plazo, las empresas de servicios turísticos invertirán en inteligencia de mercado, chatbots (servicios automatizados de mensajería instantánea), comunicación por voz y realidad virtual, a fin de generar mayores ingresos, mejorar su atención y conocer las necesidades del turista. (PROMPERU, 2019)

Las actividades y experiencias se entrelazan o interactúan con plataformas digitales, la conectividad, las actividades como el geocatching, la realidad aumentada, y los servicios que emplean SoLoMO (social location mobile), son uno de los pocos ejemplos de las aplicaciones empleadas por el turista de hoy.

Teniendo en cuenta la naturaleza multifásica de la experiencia turística: sueño, investigación, reserva, experimentación, intercambio de información (share) Agapito, Valle y Mendes (2014) sugieren que las experiencias in loco podrían mejorarse explorando el potencial de las Tecnologías de la Información y la Comunicación (TIC) en diferentes períodos durante el viaje, lo que significa que el entorno puede ser tanto físico como virtual (pp. 5-6). Los investigadores utilizan el término in loco para establecer que las acciones se desarrollan «en el lugar de», explicando que las interacciones en el mundo virtual tienen respuesta en el mundo material cuando se trata de solicitar un servicio o acondicionarlo para una optimización de la experiencia, el servicio y la atención al cliente han cambiado y seguirán en una línea que integre nuestras intervenciones en el mundo digital y las necesidades del servicio.

En un contexto donde la actividad turística se complementa con el uso de tecnología móvil el término usabilidad adquiere mayor relevancia para evaluar el uso de las aplicaciones móviles, sistemas de gestión, operación y ventas en el ámbito turístico. Son muy pocos los estudios de usabilidad y turismo, la mayoría de los estudios se orientan al desarrollo de softwares de uso turístico. 
Schwabe, Novak y Aggeler (2008) ponen a prueba el concepto de usabilidad por intermedio de un software para varias agencias de viajes, considerando como una de las principales dimensiones la satisfacción de uso y la percepción del usuario. En la evaluación general de usabilidad en la agencia de viaje Smart Travel Prototype, encontraron respuestas muy alentadoras y relevantes en cuanto a la puesta en marcha del modelo y la evaluación de su sistema bajo el criterio de usabilidad. Los resultados fueron los siguientes: interacción del sistema claro y comprensible, aprobación 93\%; fácil de aprender $88 \%$; fácil de usar $87 \%$; ayuda en la planificación del viaje $88 \%$; les gustó el uso de información multimedia $100 \%$. Los usuarios elogiaron especialmente la interacción táctil como una idea novedosa y compararon el sentido del tacto como «acercar el sentido de las vacaciones» (Schwabe et al., 2008, p. 11).

En NSAI Standard (2019) se propone que la usabilidad no debe confundirse como el atributo de un producto, así los atributos propios del producto puedan contribuir a que este sea utilizable en un contexto particular de uso. Ello nos aclara que la usabilidad tiene un enfoque más amplio y holístico para interpretar la capacidad de uso, advirtiéndonos que implica otras dimensiones.

Las múltiples dimensiones que enmarcan las experiencias turísticas se deben observar bajo el criterio de la usabilidad en el sentido de que apoderarse de la experiencia, coincide con una experiencia memorable.

En este sentido refieren que los marcos que apuntan a crear las experiencias turísticas deseadas se centran en los factores externos que influyen en la percepción de los turistas, que pueden ser organizados en parte por el destino, desde una perspectiva de gestión de marketing. Estos instrumentos enfatizan la importancia de estimular los cinco sentidos humanos de los turistas para lograr respuestas individuales positivas. (Agapito et al., 2014, p. 6)

La naturaleza de la experiencia turística está íntimamente relacionada con los sentidos, pero su estímulo depende de cada usuario o visitante, todos y cada uno tienen diferentes formas de atrapar las experiencias, y la tecnología surge como un facilitador de la experiencia. «A pesar de estar expuestos a los 
mismos estímulos, los turistas atienden diferentes elementos, lo que significa que experimentan destinos de múltiples maneras» (Agapito et al., 2014, p. 6).

\section{Usabilidad y la facilitación como mediación de la experiencia}

Las experiencias turísticas implican una dinámica centrada en la percepción de la realidad, basada en estímulos que intervienen en la mente del visitante, debido a la singularidad de cada individuo, las reacciones frente a estos estímulos y su percepción es condicionada por factores externos y otros internos que involucran el temperamento, el estado físico, las costumbres, etc. Ooi (2005), plantea:

Es más probable que un producto turístico sea apreciado de diversas maneras si los mediadores no dirigen a los turistas hacia formas limitadas o específicas de apreciación y disfrute. En su tarea de generar experiencias turísticas, los mediadores tienen que lidiar con la escasez de atención humana, así como con varias fuentes de distracción. (p. 58)

El concepto de mediación de experiencia turística da lugar, no solo a las personas, sino también a los elementos físicos, infraestructura e incluso dispositivos de ayuda que en muchos casos sirven para informar y orientar al visitante en el destino.

La mediación interviene en los contextos socioculturales, el entorno físico y las preconcepciones turísticas del producto turístico, esta última se relaciona a los hábitos, la cultura y otros elementos que intervienen en las expectativas del visitante, además los mediadores tienen un rol de facilitación de la experiencia turística y deben intervenir en todos los ámbitos de su competencia.

La mediación de las experiencias no significa el control absoluto de la atención de los turistas, sino un equilibrio a la necesidad del turista para que interprete el producto turístico de manera deseable, al tiempo que le permita sentirse comprometido en la toma de decisiones, superación de dificultades y la brecha extranjera - local (Ooi, 2005, p. 58) 
En este contexto es preciso incluir facilitadores o instrumentos de facilitación que logren los objetivos deseados como despertar y estimular la atención del visitante, reconociendo el contexto, los hábitos y las formas de interactuar bajo el criterio de usabilidad de manera intuitiva, y que ejerza una fuerza que ayude al visitante a apoderarse de la experiencia con los sentidos de manera muy sutil y fácil de usar.

Por un lado, los estímulos sensoriales integran los factores ambientales que componen el entorno en el que tiene lugar el consumo de productos (tangibles e intangibles). Por otro lado, el ambiente circundante resultante es un facilitador de las interacciones humanas entre turistas y empleados, otros turistas, y la comunidad. (Agapito et al., 2014, p. 5)

Desde el enfoque de mediación Ooi (2005) afirma que «los mediadores turísticos ofrecen un camino más corto a los turistas que carecen de información y conocimiento de las sutilezas locales, es decir, dirigen al turista hacia ciertos productos e interpretaciones de productos para que consuman un lugar» (p. 57).

Reconociendo este importante aspecto que consiste en encaminar la experiencia turística junto con el uso complejo de formas que involucran elementos físicos, como señalética, decoración, dispositivos y además la conectividad, se deben priorizar los contextos que influyen en la percepción y juicio del visitante. Como lo refieren Agapito et al. (2014), «los aspectos sensoriales de los destinos se han enfocado recientemente como una dimensión importante en el proceso de facilitar experiencias turísticas positivas» (p. 30). Es decir, deben entenderse en un sentido más amplio como se plantea en la investigación realizada por Schwabe et al. (2008):

El diseño centrado en el usuario requiere la participación del usuario desde el "primer día», el objetivo es identificar las verdaderas necesidades del usuario, como son difíciles de formular, se recomiendan pruebas de usabilidad, pruebas de campo y pruebas piloto, así como apoyo durante la implementación organizacional. Solo al ver los resultados (intermedios), los usuarios pueden juzgar si sus verdaderas necesidades están satisfechas. (p. 5) 
Los medios tecnológicos y servicios soportados en conectividad y dispositivos desarrollan la tarea de facilitación en la experiencia turística, de forma directa una asistencia remota o videollamada también es considerada parte de una experiencia turística dentro del servicio. En tal sentido, Agapito et al. (2014), plantean que los temas informados sensorialmente podrían utilizarse para organizar creativamente y comunicar experiencias turísticas, al explorar tanto la idea de la naturaleza de múltiples fases de las experiencias turísticas como el potencial de las TIC. Los Sistemas de Información Geográfica (SIG), la tecnología Global Positioning System (GPS) y otras tecnologías podrían ser adoptadas en el desarrollo de actividades de geocaching, mapas interactivos y boletines informativos, juegos publicitarios y sitios basados en información sensorial (p. 28).

La usabilidad como forma de adaptar herramientas, sistemas o servicios a una experiencia satisfactoria de uso, se hace más evidente por su contribución en el diseño de experiencias turísticas a nuevos escenarios y contextos de la experiencia turística y el servicio.

\section{Usabilidad y diseño centrado en usabilidad turística}

La palabra «usabilidad» la podemos encontrar en definiciones que se relacionan con la capacidad de usar o facilidad de uso. La norma ISO en un sentido más amplio nos ofrece una idea más técnica y apropiada del uso de este término:

El diseño centrado en el ser humano es un enfoque para el desarrollo de sistemas interactivos que tiene como objetivo hacer que los sistemas sean utilizables y útiles, centrándose en los usuarios, sus necesidades, requisitos, y aplicando factores humanos, ergonomía, conocimientos y técnicas de usabilidad. Este enfoque mejora la efectividad y la eficiencia, mejora el bienestar humano, la satisfacción del usuario, la accesibilidad y la sostenibilidad; y contrarresta los posibles efectos adversos del uso en la salud humana, la seguridad y el rendimiento. (ISO, 2019)

En la actualidad, la norma ISO 9241-11: 2019 mantiene la definición del término en su revisión del 2018 (ISO 9241-11: 2018, 3.1.1); en ella se señala hasta qué punto un sistema, producto o servicio puede ser utilizado por 
usuarios específicos para lograr objetivos específicos con efectividad, eficiencia y satisfacción en un contexto de uso específico. La palabra «usabilidad» también se usa como un calificador para referirse a los conocimientos de diseño, competencias, actividades y atributos de diseño que contribuyen a la usabilidad, como experiencia en usabilidad, profesional de usabilidad, ingeniería de usabilidad, método de usabilidad, evaluación de usabilidad, usabilidad heurística (ISO, 2019). En este contexto, es importante considerar que «la usabilidad depende de las circunstancias específicas en las que se utiliza un sistema, producto o servicio» (NSAI Standard, 2019, p. 14).

El término usabilidad, tanto en su acepción como proceso y producto nos dan como referencia la necesidad de orientar los esfuerzos para lograr que los usuarios logren sus objetivos con satisfacción, implicando un desarrollo orientado al uso o manejo por parte de ellos en un contexto que requerirá, por supuesto, de ciertos elementos y aspectos en su diseño que faciliten este manejo.

El término usabilidad como proceso se define como la eficacia, eficiencia y satisfacción con la que un sistema permite alcanzar objetivos específicos a usuarios concretos en un contexto de uso también específico según la ISO 9241-11:1998. Por otro lado, tenemos una acepción de acuerdo con la ISO/IEC 9126-1:2001 se define como la capacidad de un software de ser comprendido, aprendido, usado y que resulta atractivo para el usuario en condiciones específicas de uso. (González-Sánchez et al., 2012, p. 531).

Nielsen (1993), estudió el concepto de usabilidad encontrando aspectos multidimensionales y transversales en su aplicación, al respecto afirma que la usabilidad no es una propiedad única y unidimensional de una interfaz de usuario. Tiene múltiples componentes y se asocia tradicionalmente con estos cinco atributos:

- Capacidad de aprendizaje: el sistema debe ser fácil de aprender para que el usuario pueda comenzar rápidamente a realizar algún trabajo con el sistema. 
- Eficiencia: el sistema debe ser eficiente de usar, de modo que una vez que el usuario haya aprendido el sistema, sea posible de aplicar y obtener un alto nivel de productividad.

- Memorabilidad: el sistema debe ser fácil de recordar, para que el usuario ocasional pueda volver al sistema después de un período de no haberlo utilizado, sin tener que aprender todo de nuevo.

- Errores: el sistema debe tener una baja tasa de error, de modo que los usuarios estén subjetivamente satisfechos cuando lo usen y les guste.

- Satisfacción: el sistema debe ser agradable de usar, de modo que los usuarios estén subjetivamente satisfechos cuando lo usen, les guste. (Nielsen, 1993, p. 26)

Las primeras investigaciones sobre usabilidad nacieron de interrogantes relacionadas con la ingeniería, específicamente con el manejo de computadoras e interfaces, el término es más amplio y su extensión está orientada a otros campos de la actividad humana.

En este sentido, Nielsen (1993) afirma que los factores humanos y la ergonomía tienen un alcance más amplio que la interacción de la computadora humana, de hecho, muchos métodos de usabilidad se aplican igualmente, bien al diseño de otros sistemas complejos como a sistemas simples que no son lo suficientemente simples (p. 24). Hoy no solo el concepto se adapta, si no que la tecnología, basada en sistemas, forma parte de la vida cotidiana y se hace interactiva.

Sin duda los componentes de la usabilidad también forman parte de las dimensiones de la experiencia turística, desde el punto de vista de la experiencia turística, el aprendizaje y la satisfacción como juicio producido entre el contacto de la realidad y las expectativas.

Sobre el uso de páginas web de información en turismo, refiriéndose a las páginas analizadas encuentran que existen desafíos de usabilidad en varios aspectos, tanto en contenido como en funciones. Muchos problemas reflejan la falta de diseño centrado en el usuario e interactividad en el desarrollo del sitio web de turismo de la ciudad. Esta 
investigación también encontró que la usabilidad está estrechamente conectada con las diferencias culturales. (Zhou \& DeSantis, 2005, p. 794)

La integración de los sistemas involucrando actividades, objetos y formas de interactuar nos dibujan un escenario en donde el término usabilidad obtiene más relevancia en el ámbito de la práctica del servicio, bajo la mirada de la experiencia del cliente. La experiencia turística en este contexto involucra múltiples servicios; sin embargo, las características de individualidad de cada visitante nos obligan a enfocar esfuerzos en diseñar productos y experiencias adecuadas al usuario, con acceso y manejo pensando en el usuario.

$\mathrm{Al}$ respecto, Zhou y DeSantis (2005) refieren:

Ciertas funciones como el recorrido virtual, la galería o el mapa interactivo pueden ayudar a los turistas potenciales a desarrollar expectativas sobre lo que pueden experimentar en el destino. La interactividad y multimedia son factores clave para proporcionar experiencias virtuales. (p. 790)

Aquí tenemos un ejemplo de cómo el uso de aplicaciones móviles contribuye en la actualidad a mejorar y complementar la experiencia del cliente.

Considerando la aplicación de la usabilidad, en turismo, existen dos aspectos, el primero orientado a integrar la usabilidad al diseño creativo involucrando el contexto, tipos, segmentos de usuarios, hábitos, preferencias; todo ello, enfocado a cumplir con los criterios de usabilidad, estableciendo indicadores. El segundo, orientado al seguimiento de su implementación, monitoreo, medición, evaluación; y tomando acciones.

Bevan, Kirakowski y Maissel (1991) establecen tres puntos de vista para la medición de la usabilidad:

- Orientada al producto, que la usabilidad se puede medir en términos de atributos ergonómicos del producto.

- Orientada al usuario, que la usabilidad se puede medir en términos de lo mental esfuerzo y actitud del usuario. 
- Orientada al rendimiento del usuario, que la usabilidad se puede medir examinando cómo el usuario interactúa con el producto, con especial énfasis en la facilidad de uso del producto o aceptabilidad, si el producto se utilizará en el mundo real.

Basados en los criterios de medición de la usabilidad de Bevan, Kirakowski y Maissel (1991), las consideraciones de medición en productos y servicios en turismo deben contemplar los siguientes aspectos:

- Contexto. Referido al producto turístico -servicio- aplicación o interface para el servicio y sus cualidades basado en un enfoque de contexto de uso. Se contemplan las percepciones, cualidades generales, orientadas a conocer si cumplen con las expectativas del segmento a quien se le ofrece, y el cumplimiento del listado de necesidades encontradas como importantes para los usuarios o visitantes.

- Interacciones. Referido a la forma de interactuar con el usuario, a la actitud, facilidad de comunicación con el usuario, percepción de las características visuales, la predictibilidad, el nivel de manejo intuitivo, y todo lo relacionado a la interacción con el medio, dispositivo y servicio.

- Indicadores. Referido a la capacidad de generar indicadores, la capacidad de definir la medición cuantitativa de las interacciones del usuario, el rendimiento, cumplimiento de las expectativas, así como la analítica de otros factores y relaciones del producto con el usuario.

Por otro lado, López (2014) indica que en el apartado 7.1 de la norma ISO 13407, existen cuatro tipos de actividades centradas en el usuario que es conveniente integrar durante un proyecto de desarrollo de un sistema. Estas actividades son:

1. Comprender y especificar el contexto de uso.

2. Especificar los requisitos referentes al usuario y a la organización.

3. Producir soluciones de diseño.

4. Evaluar los diseños respecto a los requisitos. (p. 25) 
De acuerdo con estas actividades planteadas para la usabilidad, la siguiente es una forma de integrar este concepto en el diseño de productos y servicios turísticos:

- Integración del contexto en la operación turística orientada al gestor u operador, así como al usuario, principal protagonista.

- Segmentación de usuarios y establecimiento del contexto de uso para cada grupo, sus preferencias y necesidades.

- Diseño de soluciones para integrar tecnología como facilitador al usuario, centrado en la experiencia del cliente en turismo.

- Desarrollo de prototipos -test de prototipos- orientados a la experiencia del usuario en turismo.

- Generación de indicadores centrados en el usuario facilitando la mejora continua del producto o servicio.

- Gestión de la mejora continua, como la capacidad de analizar y tomar acciones correctivas, solucionar y mitigar posibles problemas encontrados, maximizar la experiencia y evitar impactos negativos.

El enfoque de la usabilidad en turismo debe considerar al cliente-usuario, representado por el visitante o turista; al operador turístico, representado por quien otorga el servicio directamente, ya sea física o por medio de un dispositivo; y de modo indirecto, a los proveedores que hacen posible el producto turístico o servicio, involucrando a los actores en un contexto de uso previamente determinado, en donde todos participan interactuando con un diseño que los involucra bajo el criterio de la usabilidad.

Con respecto a los beneficios de la usabilidad en el contexto del diseño turístico, Zhou y DeSantis (2005) afirman:

La riqueza de la información sensorial y la interactividad entre el entorno humano y virtual son dos factores cruciales para la planificación del viaje y concluyeron que un recorrido virtual puede mejorar la memoria del destino y puede ayudar a los usuarios a formar una imagen de destino más vívida y clara. (p. 790) 
Por consiguiente, la usabilidad debe cumplir un rol facilitador y orientador. De este modo, la usabilidad se centra en el cliente o visitante y también en el operador bajo un enfoque de cliente interno, pues este último es quien hace posible que se brinde el servicio. Ambos actores participan de un diseño orientado a la entrega de experiencias bajo el criterio de usabilidad que le otorga al servicio memorabilidad y una vigencia como producto en la preferencia de la demanda.

La búsqueda por diseñar un producto o servicio con una mirada integral orientada a los requerimientos del visitante o turista, en donde el conocimiento es la clave del proceso de diseño en un ciclo dinámico.

El proceso parte de la comprensión del contexto y la verdadera necesidad del visitante, conociendo sus necesidades, razones por las que toma el servicio, siguiendo un recorrido que busca entender los tipos de usuarios o clientes para una búsqueda creativa de soluciones y modelos, a los que luego, se le añadirán indicadores que produzcan información y nuevo conocimiento que ayude a plantear mejoras en el siguiente proceso y una mejor comprensión del contexto en el diseño de la experiencia turística.

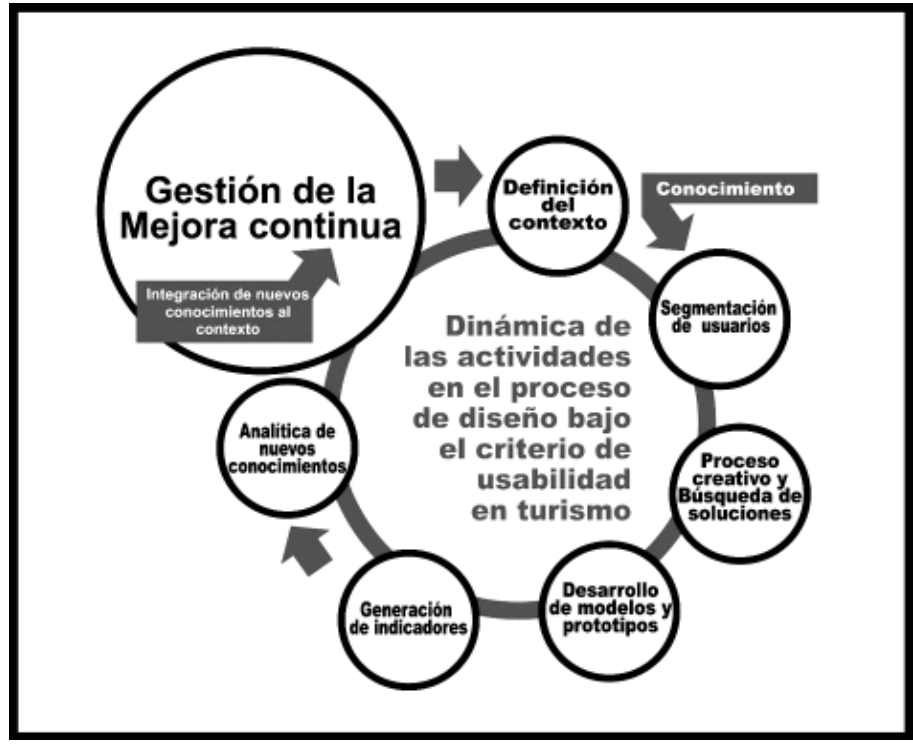

Figura 1. Proceso de diseño en un ciclo dinámico bajo el concepto de mejora continua y criterios de usabilidad. 
El recorrido del conocimiento en el diseño nos ayuda a comprender con más claridad esta dinámica que integra el criterio de usabilidad, es así que el proceso se inicia con la definición del contexto o problema a solucionar, la comprensión de quiénes son los involucrados con la segmentación de usuarios, identificando tanto al cliente interno como externo; de este modo, obtenemos un idea más clara de lo que debemos hacer y para quién lo estamos haciendo, una vez que hemos llegado a este punto entendiendo a quiénes nos dirigimos y cuál es el problema, planteamos de forma creativa soluciones.

Como siguiente paso, en este proceso se desarrollan modelos y prototipos identificando las preferencias y cualidades que el usuario busca encontrar en nuestro diseño, para luego generar indicadores que hagan posible la medición de la satisfacción y percepción de los usuarios. Lo que continúa, igual de importante es analizar la información obtenida luego de la prueba o simulación del modelo generando nuevo conocimiento que se pondrá en marcha para retroalimentar nuestro modelo. Es decir, añadiendo nuevo conocimiento al contexto y al conocimiento del cliente frente a la experiencia desarrollada, logrando un producto mejorado, ya sea en el ámbito de la exploración de nuevos prototipos como en diseños que se están explotando en el ámbito comercial.

\section{Conclusiones}

Las aplicaciones tecnológicas han generado múltiples cambios en los diversos ámbitos a lo largo de la historia humana. El turismo como actividad involucra diversos actores de manera transversal y diferentes actividades que posibilitan su gestión; aprovecha de manera significativa el impacto de la conectividad, la inteligencia artificial, el almacenamiento en la nube y el «internet de las cosas» (IoT, por sus siglas en inglés).

Existe evidencia de que el reciente impacto de las tecnologías de la información y la comunicación, han cambiado profundamente la naturaleza de las experiencias turísticas, por tanto, su comprensión es crucial para crear y entregar experiencias competitivas en el futuro.

El impacto en las condiciones que mejoran la calidad de vida y la forma como pueden ayudar en la conservación del medio ambiente y el uso racional 
de los recursos es una tendencia sobre el manejo de la tecnología. En este sentido, Sanabre, Vinyals-Mirabent y Pedraza-Jiménez (2019, p. 8) sostienen que desarrollar la presencia virtual mediante sitios web de éxito es uno de los retos principales de las llamadas Organizaciones de Marketing de Destino (OMD) y los Destinos Turísticos Inteligentes (DTI).

La mirada centrada en el usuario es la clave para el desarrollo de esta nueva tecnología en los próximos años, así como también el consenso de las empresas para desarrollar estándares que beneficien y otorguen valor al usuario. Rose, Eldridge y Chapin (2015) afirman que «el Internet de las Cosas está ocurriendo ahora, y promete ofrecer un mundo revolucionario, 'inteligente' y totalmente conectado a medida que las relaciones entre los objetos, su entorno y las personas estén cada vez más entrelazadas» (p. 8). Es así que el concepto de usabilidad como facilitador de la experiencia turística debe ser un criterio que se debe tomar en el diseño de experiencias turísticas.

El diseño de experiencias bajo el criterio de usabilidad como facilitador debe implicar tres niveles de usuarios, el primero, relacionado al protagonista principal que es el visitante o turista; el segundo, a quien o quienes entregan el servicio y están en contacto con el visitante o turista; y el tercero, dirigido a quienes integran la operación de manera indirecta y son el soporte importante en la gestión de la experiencia del cliente.

El criterio de usabilidad en las aplicaciones y procedimientos que se diseñen, debe incluir los siguientes indicadores, en sus tres niveles de usuarios:

Indicadores del criterio de usabilidad en un proceso, servicio, o aplicación:

- Facilidad de usar, manejar

- Manejo intuitivo, de fácil razonamiento o deducción para manejar

- Fácil de aprender

- Facilidad de retener el manejo en la memoria

- Capacidad de comunicar (audio-video - imágenes)

- Capacidad de compartir (share)

- Personalizable 
- Multi-lenguaje y uso de iconos y símbolos universales

- Ayudas y referencias

Indicadores del criterio de usabilidad relacionados a dispositivos $\mathrm{u}$ objetos (elementos físicos):

- Relacionados al hábito de los usuarios, a experiencias de manejo de otros dispositivos similares, o hábitos de costumbre

- Impactos psicológicos y de comportamiento

- Elementos estéticos

- Maniobrabilidad de las características físicas

Indicadores del criterio de usabilidad relacionados a los sentidos:

- Uso táctil, entorno visual, audio

- Portabilidad, de fácil interacción con otros dispositivos

- Transmisión y almacenamiento de información

- Velocidad

- Seguridad y privacidad

Los criterios deben otorgar una adecuada experiencia al usuario y fomentar el hábito de uso, como resultado de la satisfacción del usuario, por otro lado, deben permitir la evaluación de los usuarios, así como la posibilidad de transmitir experiencias y consejos. Los criterios se deben manejar en niveles, fáciles de cuantificar para su posterior análisis y comprensión.

El criterio de usabilidad en el turismo contempla:

- Elementos utilitarios: sentido de utilidad y eficiencia en la entrega de valor en el servicio.

- Habitos y multiculturalidad: adaptación a los hábitos del usuario (facilidad de configurar opciones de usuario y operador, orientado para ser manejado por diferentes tipos de usuarios, configuración personalizada). 
- Facilidad de aprendizaje: uso memorable (fácil o adecuado nivel de recuerdo y memorización, fácil de hacer y repetir). Intuitivo (facilidad de interpretación, uso de símbolos convencionales).

- Retroalimentación: interacción entre partes, permite al usuario comunicar al operador sus necesidades o informar con su comportamiento, advierte necesidades y corrige procesos.

- Uso de tecnología y conectividad: se trata de comunicar personas, objetos, dispositivos, etc.; muchos canales que permitan la toma de decisiones y el óptimo uso de los recursos en el marco de la sostenibilidad, inclusión y multiculturalidad.

- Ergonomía: adaptado a la condición humana, buscando la eficiencia del servicio, bajo una lógica de uso predictivo, simple, visual a través de los sentidos.

\section{Referencias}

Agapito, D., Valle, P., \& Mendes, J. (2014). The sensory dimension of tourist experiences: Capturing meaningful sensory-informed themes in Southwest Portugal. Tourism Management, 42, 224-237.

Bevan, N., Kirakowski, J., \& Maissel, J. (1991). What is Usability? Recuperado de https://www.researchgate.net/ publication/239609271_What_is_Usability

González-Sánchez, J. L., Montero-Simarro, F., \& Gutiérrez-Vela, F. L. (2012). Evolución del concepto de usabilidad como indicador de calidad del software. El profesional de la información, 2012, septiembreoctubre, 21(5), 529-536. http://dx.doi.org/10.3145/epi.2012.sep.13

ISO. (2019). ISO 9241-210:2019. Recuperado de https://www.iso.org/obp/ui/es/\#iso:std:iso:9241:-210:ed-2:v1:en

López, M. A. (2014). Desarrollo de una aplicación web centrada en el usuario tomando como guía la norma ISO 13407 que permita superar problemas presentados en una institución que cuenta con una aplicación desarrollada tradicionalmente (Tesis de maestría). Pontificia Universidad Católica, Perú.

Neuhofer, B., Buhalis, D., \& Ladkin, A. (2014). A typology of technology enhanced tourism experiences. International Journal of Tourism Research, 16(4), 340-350.

Nielsen, J. (1993). Usability Engineering, Academic Press, Inc., USA.

NSAI STANDARD. (2019). Ergonomics of human-system interaction - Part 11: Usability: Definitions and concepts (ISO 9241-11:2018) Irish Standard ISO. Recuperado de https://infostore.saiglobal.com/preview/is/en/2018/ i.s.enis09241-11-2018.pdf?sku=1980667

OMT Press Release. (2019). Google se asocia con la OMT para avanzar en el desarrollo de competencias digitales PR 19018 Organización Mundial del Turismo OMT. Www2.unwto.org. Recuperado de http:// www2.unwto.org/es/press-release/2019-03-07/google-se-asocia-con-la-omt-para-avanzar-en-el-desarrollode-competencias-d 
Ooi, Can-Seng. (2005). A theory of tourism experiences: The management of attention. Experiencescapes: Tourism, Culture and Economy, 51-68. Recuperado de https://www.researchgate.net/publication/ 284036434_A_theory_of_tourism_experiences_The_management_of_attention

PROMPERU. (2019). El impacto de los smartphones durante los viajes, turismo In Boletín № 54 - Febrero. Recuperado de https://www.promperu.gob.pe/turismoin/Boletines/2019/feb/5_el_impacto_de_los_ smartphones_durante_los_viajes.html

Rose, K., Eldridge, S., \& Chapin, L. (2015). La internet de las cosas-una breve reseña. Internet Society (Ed.). Recuperado de https://www.internetsociety.org/wp-content/uploads/2017/09/report-InternetOfThings20160817-es-1.pdf

Sanabre, C., Vinyals-Mirabent, S., \& Pedraza-Jiménez, R. (2019). Calidad de los sitios web turísticos. El caso del DTI Barcelona. ARA: Revista de Investigación en Turismo, 9(1), 7-17.

Sakulsureeyadej, A. (2011). Cómo la tecnología está cambiando el turismo mundial. AM reports vol.1, Tecnología en el turismo, OMT. Recuperado de https://www2.unwto.org/sites/all/files/pdf/omt_amreports_numero1_ tecnologiaturismo_esp.pdf

Schwabe, G., Novak, J., \& Aggeler, M. (2008). Designing the Tourist Agency of the Future. BLED 2008 Proceedings, 42.

Zhou, Q., \& DeSantis, R. (2005). Usability issues in city tourism Web site design: a content analysis. In IPCC, Proceedings. International Professional Communication Conference, 2005 (pp. 789-796). IEEE. doi: 10.1109/ IPCC.2005.1494253 